\title{
CONTROLLABLE LOAD FOR A MICROGRID TESTBED
}

\author{
John Konstantaras*, \\ llias Billas,

\section{Eleftherios Tsambasis}

Energy Systems Laboratory, National \& Kapodistrian University of Athens, Athens, Greece

\section{Correspondence:}

John Konstantaras

e-mail:

john.konstantaras@teiste.gr

\begin{abstract}
:
Microgrids are a building block of the smart grid facilitating the renewable energy sources penetration and customer involvement in demand side management. As the transition from the conventional to the smart grid progresses, the need for appropriate tools for development and testing increases as well. In this work we propose a design for a controllable load to be used with a microgrid testbed used to study stability, sizing and power quality aspects of microgrids. The smart load consists of commonly used loads controlled by a programmable logic controller to emulate actual load curves of a university building. It has been tested for a period of over a year using an existing autonomous microgrid that consists of photovoltaic panels and batteries. The results demonstrate its capability to emulate successfully actual load curves and its potential in microgrid testing.
\end{abstract}

\section{Keywords:}

smart load, hybrid microgrid, storage, load curve emulator.

\section{INTRODUCTION}

As the shift towards the smart grid [1] progresses, and the technology for renewable energy source (RES) integration improves, microgrids are viewed as an essential building block of the grid at the distribution level. The microgrid is a small-scale electrical power localized system which operates in grid-connected or island mode, may connect to the grid at the Point of Common Coupling (PCC), uses distributed energy resources (DERs), with or without storage, and serves local loads. Microgrids are expected to facilitate RES penetration, improve the grid's stability, resilience and quality of service and enable customer involvement in demand side management technologies [2,3].

A hybrid microgrid consists of various types of DERs, renewable or conventional [4]. DERs may be controllable, e.g. a fuel cell or a generator, or uncontrolled, e.g. PV panels or wind turbines. Hybrid microgrids with storage seem to be the most promising candidate for the emerging paradigm of the smart grid with high RES penetration. However, the storage technology lags behind the microgrid requirements for safety, cycle life, energy density, and cost [2]. 
Microgrid testbeds can be used to experimentally test cases related to efficiency, optimal sizing and control, quality of service and resilience. To address these problems a controllable load is necessary to emulate the consumption profile of an entity, be it a user, a group of users or a microgrid. In the literature the term smart or controllable load usually refers to devices that can be controlled by a SCADA [5] or a building management system, such as thermostats [6] or "smart plugs". In this work, we use the term to describe a load that can emulate any given consumption profile.

The proposed controllable load presented is powered by a hybrid autonomous microgrid that serves as a testbed for the study of aspects of optimal microgrid design [7] and demand side management [8]. It is designed to emulate the actual load curve of a University building for various days of the year, scaled down to the capacity of an existing microgrid consisting of photovoltaic panels (PVs) and lead-acid batteries (Fig.1).

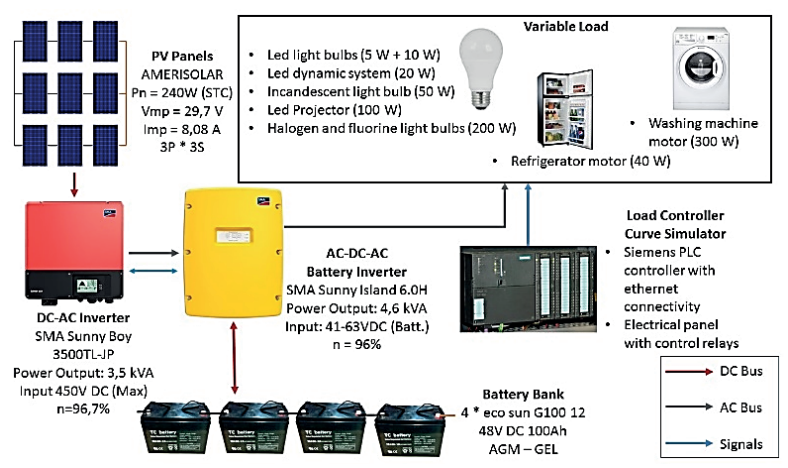

Fig. 1. The microgrid testbed with the controllable load

In the following section we discuss the requirements for the controllable load as dictated by the microgrid and the load studied. In section III, the system design and implementation is presented and section IV presents and discusses the test results.

\section{SYSTEM REQUIREMENTS}

The microgrid that will serve the controllable load is installed at the Euripus campus of the National and Kapodistrian University of Athens (NKUA) in Evia, Greece. The load curves emulated are those of the main University building. The daily profile varies significantly with the day of the week and the time of the year. The highest consumption is observed during exam weeks in January and June, which are also months of very low and very high temperatures, respectively, while the lowest consumption, which can be as low as one-third of the maximum value, is observed on weekends, holidays and vacation periods, regardless of the season. The minimum, average and maximum power is $2.00 \mathrm{~kW}, 147.13 \mathrm{~kW}$ and $529.40 \mathrm{~kW}$ respectively while the minimum, average and maximum daily electricity consumption is $1434.00 \mathrm{kWh}$, $3531.16 \mathrm{kWh}$ and $8086.40 \mathrm{kWh}$ respectively.

The area is characterized by strong solar irradiance, with Global Horizontal Irradiance, GHI $=1682 \mathrm{kWh} / \mathrm{m} 2$ per year, and PV expected electricity output, $\mathrm{PVout}=$ $1495 \mathrm{kWh} / \mathrm{kWp}$ but has a negligible wind field, less than $300 \mathrm{~W} / \mathrm{m}^{2}$ with velocities less than $5 \mathrm{~km} / \mathrm{h}$.

Due to the low wind power capacity, the microgrid relies almost entirely on solar power, featuring $2160 \mathrm{Wp}$ PVs and 400Ah battery storage. A 400W WT and a $3 \mathrm{~kW}$ generator are also installed but they haven't been used in the tests discussed in this work. The microgrid has the capability for grid-tied operation but the results reported here have been obtained in island mode operation. The generation and storage systems are controlled by a commercial AC/DC/AC inverter with rated output power of $4.6 \mathrm{kVA}$ and efficiency rating over $95 \%$.

Sensors, connected to the controller/inverter unit, measure the DC current generated by the PVs, the temperature of air, PV panels and batteries, the wind velocity and the adjacent radiation on the PV panels.

Typical load curves that reflect the characteristic operation of a university building at different times of the year have been selected from available hourly data of the main university building. As is the case with university buildings, the power consumption is a function of the weather conditions as well as the day of the week or period of operation. For example, the average and peak load of an exam day in June may be three times as much of an equally hot August day during the vacation period. In this work, to prove the flexibility and scalability of the proposed controllable load, we show the results obtained for 4 types of curves reflecting the average hourly consumption of 4 months: January, April, July and October. The data granularity necessary for the controllable load was set to 30 minutes. To double the granularity of the available data, interpolation method was used between existing data points. Given that the peak load is over $500 \mathrm{~kW}$, the load curves were scaled down by a factor of 0.0035 to match the generation capacity of the microgrid (Fig. 2). 


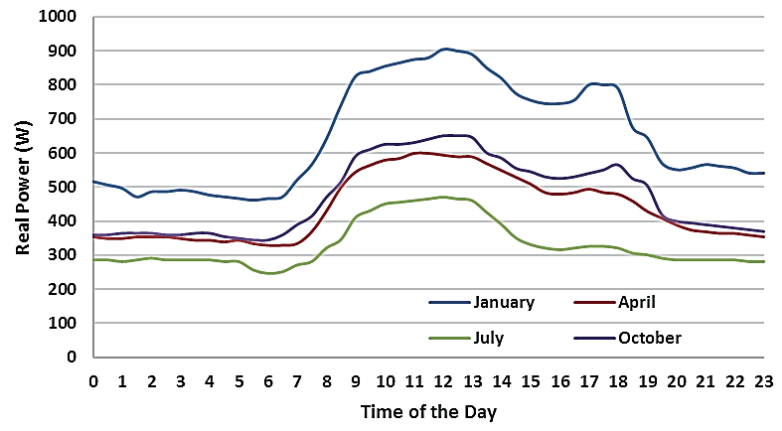

Fig. 2. The four load curves to be emulated by the controllable load correspond to the average hourly consumption of January, April, July and October scaled down by a factor 0.0035

The month of January requires the highest amount of electrical energy because of the lighting and heating needs as well as the long hours of operation, reflected especially in the afternoon peak. This load curve will test the boundaries of the microgrid's operation as it corresponds to a month of relatively low solar irradiance and low expected generation.

The April load curve corresponds to medium consumption with a smooth differential consumption profile which reflects the mild weather of the season, the longer days, and the typical class attendance. This is a less demanding curve for the microgrid to serve.

The July load curve is a low consumption curve since for the biggest part of it there are no classes due to summer holidays. July is also a month of high solar irradiance and very long days, so the generation is expected to be in the highest levels. This curve will also allow the study of the effect of the high temperatures recorded on location during the summer, which are expected to challenge the system.

The load curve of the month of October is similar to that of April with the exception of the shorter duration of days which reflects in the secondary peak observed in the evening hours. The solar irradiance is also lower, not only because of the earth's location with respect to the sun but also because of the rainy weather in that season.

The next step was to set the requirements for the distinct actual loads that will be used to emulate the scaled down load curves. First, we determine the base load of each curve and then use combinations of eight different devices to obtain the remaining (variable) load. The load is assumed to be constant for each 30-minute interval, the minimum allowed variation is $5 \mathrm{~kW}$ and its actual (scaled down) value is rounded to the nearest permissible value.
The 30-minute interval values of each one of the four load curves are shown in Fig. 3.

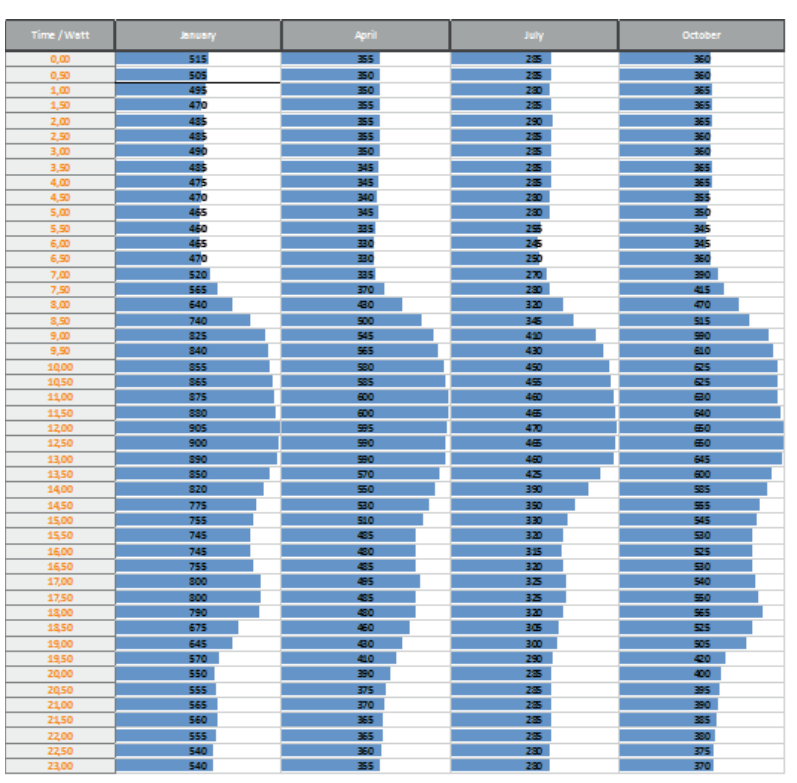

Fig. 3. The four load curves emulated by the controllable load correspond to the average consumption of January, April, July and October

\section{EXPERIMENTAL SETUP}

The base load of each month was emulated by a purely resistive load of $445 \mathrm{~W}, 320 \mathrm{~W}, 240 \mathrm{~W}$ and $325 \mathrm{~W}$ for January, April, July, and August respectively. The variable part of the load was constructed by washing machine and refrigerator motors and various types of light bulbs. More specifically, we used a refrigerator adjustable motor set to $40 \mathrm{Watt}$ using an auxiliary resistor, a washing machine motor regulated to 300 Watt with controllable speed using a transformer and a resistor, $5 \mathrm{~W}$ and $10 \mathrm{~W}$ led lighting, dynamic $20 \mathrm{~W}$ led lighting, a 50W incandescent light bulb, a $100 \mathrm{~W}$ led video projector, and 200W halogen and fluorescent light bulbs. The selection of loads was such as to allow the study of power quality problems not discussed in this work.

The variable load is controlled by relays driven by a 240W, 24Vdc Siemens S7-300 PLC with a (312-1AE140AB0) CPU, 16 digital 24Vdc inputs (321-1BH02-0AA0) and 16 digital $24 \mathrm{Vdc}$ outputs (322-1BH01-0AA0) (Fig. $4)$. 

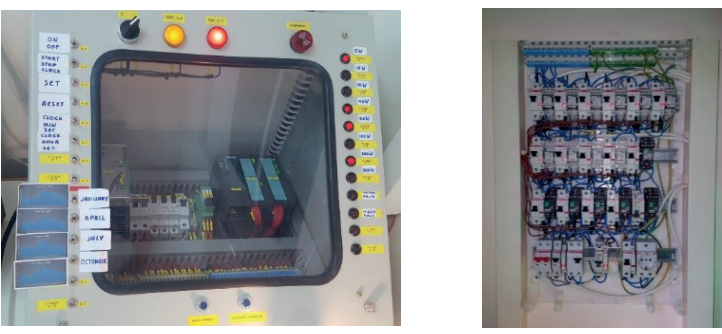

Fig. 4. The PLC and distribution panel of the system

The PLC is programmed to read the desired load for every 30-minute interval for a given month, calculate the appropriate combination of loads and activate / deactivate loads accordingly. Indicator lights inform the user of which loads are active at each given time, and the power supply from the microgrid inverter. The system also allows for an external power supply, e.g. the grid, which is not implemented in the current configuration. All results shown here have been obtained using the power generated by the RES microgrid in off grid mode.

\section{RESULTS AND DISCUSSION}

An Efergy ${ }^{\circ}$ smart meter and a SIMEAS P power analyzer were used to monitor the consumption of the system. Several tests were performed for each load curve at different days under various weather conditions. For each test, the batteries were fully charged before turning on the load.

To test the impact of weather conditions, all four curves were tested under different weather conditions. For tests run during the winter season when the hours of daylight and solar irradiance are less and cloudy and rainy days are more often, the January and October curves had the highest number of "blackouts" especially during the night. The productivity of electrical power from the panels was less than in the spring period and the batteries couldn't supply the load during the nighttime. The July curve fared better because of the low energy demand and energy increments. However, for tests run during the spring season, the number of failures was the smallest for all curves, as the sunny hours were more and the weather was better. The summer tests verified our expectations for lower PV performance and higher losses due to the high temperatures.

Figure 5 shows curves obtained from the smart meter interface for cases that the microgrid failed to supply the load even when the base load was decreased to $50 \mathrm{~W}$ to facilitate its operation during the winter nights. The top graph shows the results of a test run on a December day, a day with low solar irradiance, using the summer curve which corresponds to the lightest load. The system runs out of power during the early hours of the morning, restarts at 07.00 , after sunrise, and runs smoothly for the rest of the day. The bottom graph of Fig. 5 shows the results of a test run on a February day, which is a day of relatively low solar irradiance, using the medium consumption spring curve. The microgrid shuts down shortly after 22.00 and unsuccessfully attempts to restart three times until early in the afternoon.

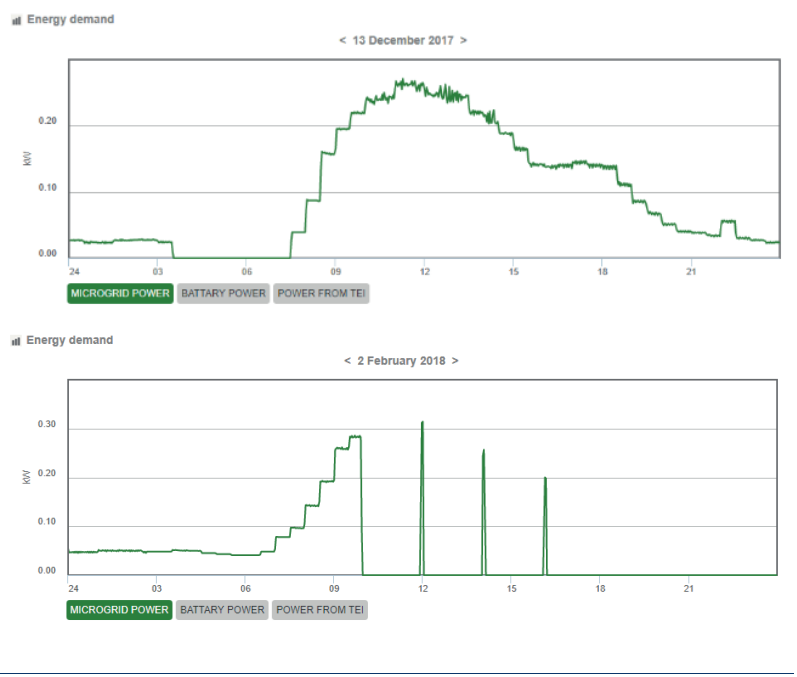

Fig. 5: Failure to supply the load

In Figure 6, the consumption of the controllable load is compared against the actual average load data for each month, for successfully completed tests where the energy supplied by the PVs and the batteries met successfully the demand of the load.

The performance of the controllable load is limited by the power granularity of $5 \mathrm{~W}$ i.e. the smallest load and the time granularity of 30-minute interval chosen.

Another problem faced during the tests run for more than a year, was the deterioration of the battery performance due to the repeatable discharging and recharging. Optimal battery sizing and performance is an important issue that all autonomous microgrids must address to curtail replacement costs. During the winter season, solar power cannot meet the load requirements and it is necessary to have alternative supplementary energy sources such as wind turbines, if the winds in the region allow it, controllable sources, such as fuel cells or microturbines, or sources with predictable performance, such as tidal generators $[9,10]$. 

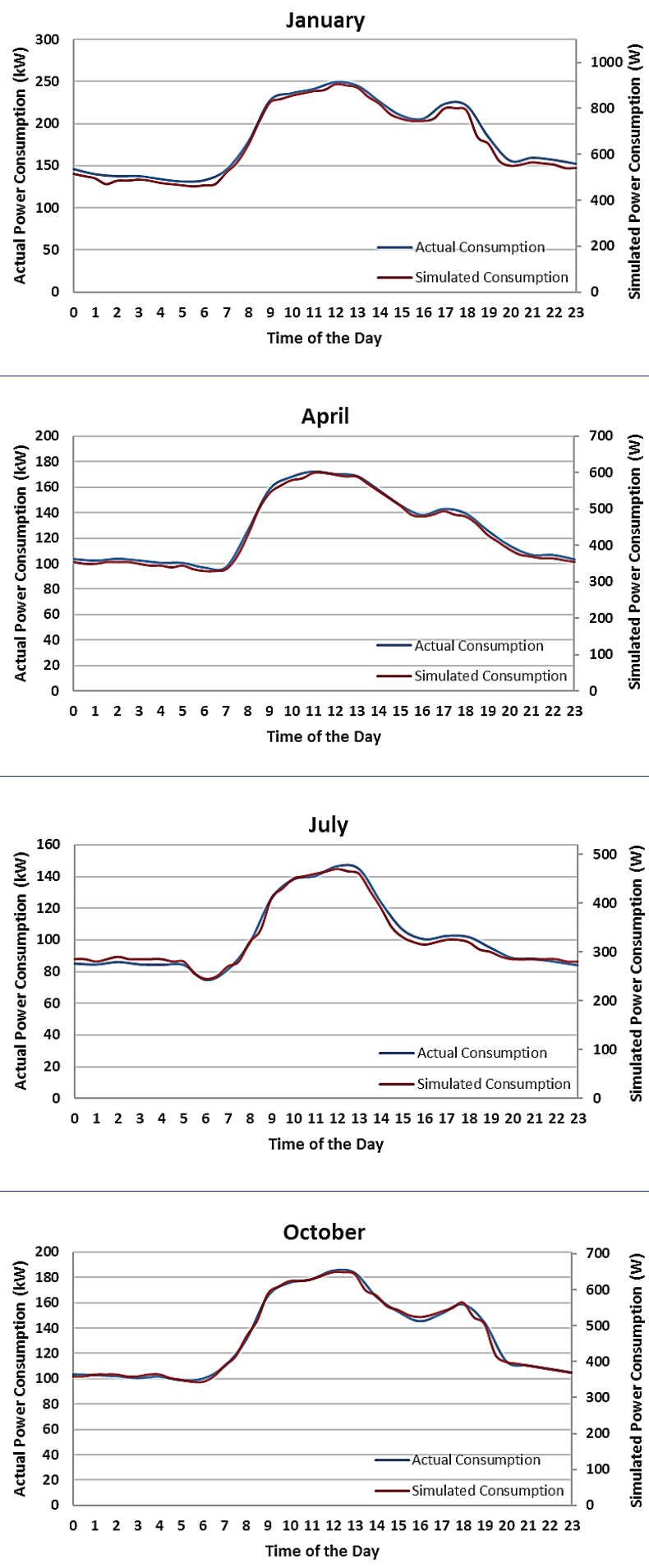

Fig. 6. Comparison between actual

(hourly averages) and emulated load curves.

In figure 7 the relative error between the actual load curve and the simulated load curve for each month is shown. The study of the simulation errors is important as it verifies the produced simulation results but at the same time enables us to expose the witnesses of the system and proceed with the necessary upgrades.
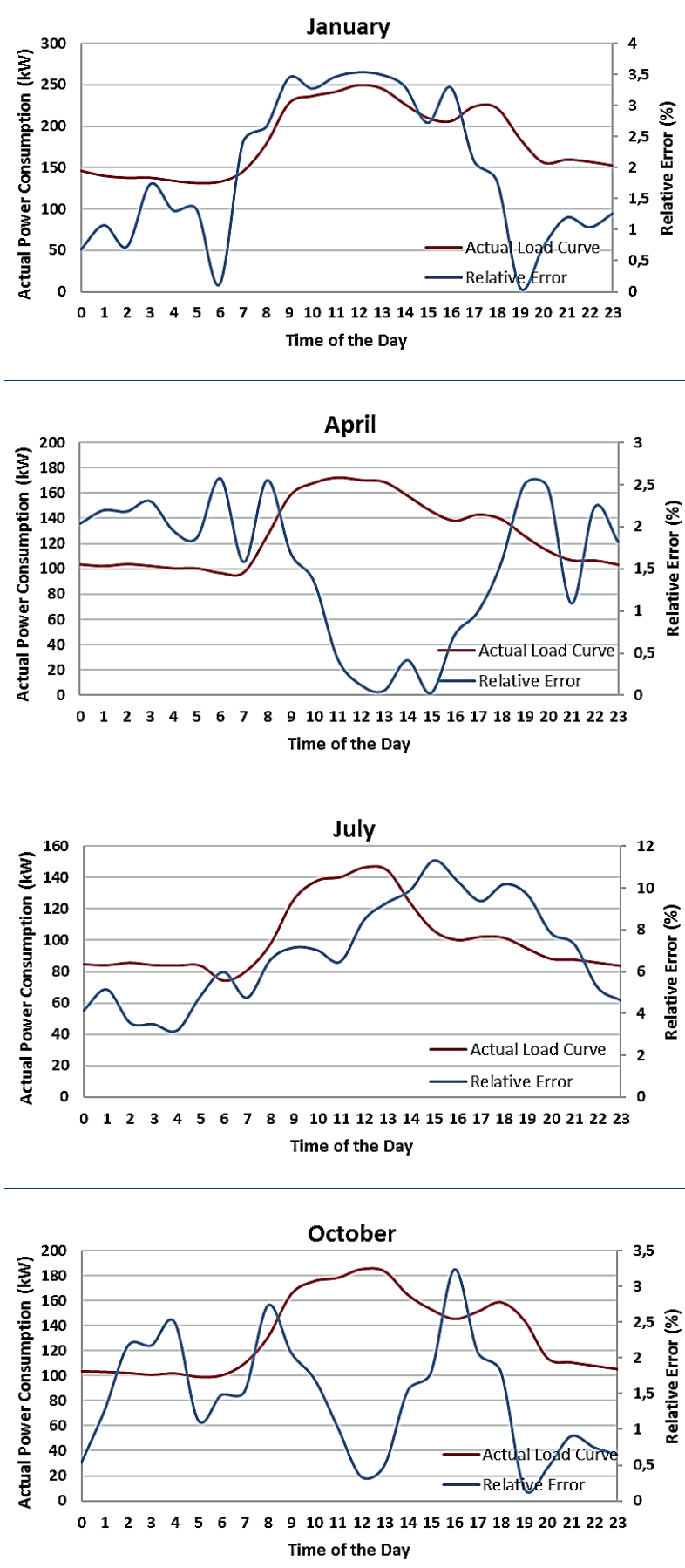

Fig. 7. Comparison between actual (hourly averages) and relative error with the emulated load curves.

For the load curve of January, the relative error between the actual and simulated curve is at an average of $1.95 \%$. The maximum error is $3.54 \%$ and appears when the load curve is at its daily peak. One the other hand when power consumption decreases so does the relative error which has a minimum value of $0.06 \%$.

The simulation of the April load curve features the lowest average error indicating that the chosen load 
values have the best fit for this load profile. The average error is $1.53 \%$. The maximum error is $2.58 \%$ and appears twice in the 24-hour period, at times when the differential load, eg the difference between two consecutive hourly loads, is highest. Greater load granularity would help the system compose a better fitted load for these times of the day and thus lower error would be exhibited. The lowest error value observed is $0.02 \%$ and occurs at times when the load is high.

The simulation of the July curve results in the highest error between actual and simulated values. The average error for this curve is $6.90 \%$ with $11.30 \%$ and $3.18 \%$ maximum and minimum values, respectively. The actual load curve for this month exhibits the lowest load among the load curves studied. From the results it is clear that greater load granularity with the addition of smaller loads will be required to better emulate such light load curves. Currently, the lightest load used in the controllable load setup described is $5 \mathrm{~W}$. To better fit the scaled down July curve, loads of $2 \mathrm{~W}$ or even $1 \mathrm{~W}$ need to be used.

The month of October has many similarities to the month of April. The average error is $1.44 \%$ with maximum and minimum values of $3.23 \%$ and $0.17 \%$, respectively. The error curve has no correlation to the load curve in terms of minimum and maximum values and it can be attributed to the load granularity used in these tests.

\section{CONCLUSIONS}

The controllable "smart" load presented in this work is designed based on requirements obtained from actual load curves to be served by a specific microgrid testbed. It is scalable and low cost as it requires commonly used loads and relays and low processing power. It will be used to study various microgrid configurations, control strategies and power quality issues.

The time and power granularities used are acceptable for studying the above-mentioned cases. In cases where the highest and lowest load curves vary greatly, better load granularity should be used to keep the error values low. Should lower errors be required, the granularity can be easily increased with the use of complimentary loads. Furthermore, the addition of smaller loads on the system would have a positive effect when power quality issues are studied.

\section{REFERENCES}

[1] H. Farhangi, "The path of the smart grid," IEEE Power and Energy Magazine, vol. 8, no. 1, pp. 18-28, January-February 2010. doi: 10.1109/MPE.2009.934876.

[2] F. Sissine, "DOE's Office of Electricity Delivery and Energy Reliability (OE): A Primer, with Appropriations for FY2016", Congressional Research Service, February 2016

[3] B. Kroposki, R. Lasseter, T. Ise, S. Morozumi, S. Papathanassiou, and N. D. Hatziargyriou, "Making microgrids work," IEEE Power and Energy Magazine, vol. 6, no. 3, pp. 40-53, May-June 2008. doi: 10.1109/MPE.2008.918718

[4] M. Patterson, N. F. Macia, and A. M. Kannan, "Hybrid Microgrid Model Based on Solar Photovoltaic Battery Fuel Cell System for Intermittent Load Applications", IEEE Transactions on Energy Conversion, vol. 30, no. 1, pp. 359-366, March 2015.

[5] F. Fernandes, T. Sousa, P. Faria, M. Silva, H. Morais, and Z. A. Vale, "Intelligent SCADA for Load Control", 2010 IEEE International Conference on Systems, Man and Cybernetics, 2010. doi: 10.1109/ ICSMC.2010.5641983

[6] J. L. Mathieu, "State Estimation and Control of Electric Loads to Manage Real-Time Energy Imbalance", IEEE Transactions on Power Systems, vol. 28, no. 1, pp. 430-440, February 2013.

[7] J. Konstantaras, Y. Koutsoubis, A. Ktena and C. Manassis, "Intelligent grid-interactive single-phase inverter", 2018 IEEE 59th International Scientific Conference on Power and Electrical Engineering of Riga Technical University (RTUCON), November 2018. doi: 10.1109/RTUCON.2018.8659831

[8] E. Mele, A. Ktena and C. Elias, "Electricity use profiling and forecasting at microgrid level", 2018 IEEE 59th International Scientific Conference on Power and Electrical Engineering of Riga Technical University (RTUCON), November 2018. doi: 10.1109/ RTUCON.2018.8659866

[9] H. Kontoyiannis, M. Panagiotopoulos, and T. Soukissian, "The Euripus tidal stream at Halkida/Greece: a practical, inexpensive approach in assessing the hydrokinetic renewable energy from field measurements in a tidal channel", Journal of Ocean Engineering and Marine Energy, vol. 1, no. 3, pp 325335, August 2015. doi: 10.1007/s40722-015-0020-8

[10] A. Ktena, C. Manasis, D. Bargiotas, V. Katsifas, Takvor Soukissian, Harilaos Kontoyiannis, "Estimation of the Energy Potential of the Euripus' Gulf Tidal Stream Using Channel Sea-surface Slope", International Journal of Monitoring and Surveillance Technologies Research, vol. 3, no. 4, pp. 23-42, October 2015. 